Submitted: 25.10.2018 Accepted: 19.11 .2018

Published: 31.12.2018

Keywords

Doppler ultrasound, renal artery, stenosis, practice guideline

\section{Recommendations for ultrasonographic assessment of renal arteries}

\author{
Anna Drelich-Zbroja', Maryla Kuczyńska1, Łukasz Światłowski \\ Anna Szymańska1, Michał Elwertowski², Agnieszka Marianowska³ \\ ${ }^{1}$ Department of Interventional Radiology and Neuroradiology, Medical University of Lublin, \\ Lublin, Poland \\ ${ }^{2}$ Department of Diagnostic Imaging, the Second Faculty of Medicine, Medical University \\ of Warsaw, Warsaw, Poland \\ ${ }^{3}$ Department of General Surgery and Endocrinology, Medical University of Warsaw, Poland \\ Correspondence: Anna Drelich-Zbroja, MD, PhD, Department of Interventional Radiology \\ and Neuroradiology, Medical University of Lublin, Jaczewskiego 8, 20-954 Lublin, Poland; \\ e-mail: zbroanna@interia.pl
}

DOI: $10.15557 / J o U .2018 .0049$

\begin{abstract}
The present clinical practice recommendations are addressed to physicians of all specialties, who perform Doppler ultrasound examinations of the kidneys on a daily basis, and in particular to specialists in radiology and imaging diagnostics. The recommendations were based on the Ultrasonography Standards of the Polish Ultrasound Society and current scientific reports consistent with Evidence Based Medicine. The paper discusses patient preparation protocol, examinat ion technique with particular emphasis on patient's position allowing to obtain proper Doppler angle of insonation, as well as diagnostic limitations of the technique. Normal blood flow parameters as well as those indicating hemodynamically significant stenosis are also presented. Although the 2013-2014 American guidelines for renal artery duplex sonography (AIUM Practice Parameter for the Performance of Native Renal Artery Duplex Sonography and AIUM Practice Guideline for the Performance of an Ultrasound Examination of Solid-Organ Transplants), which were the basis for many national recommendations, have not been significantly updated to date, a large body of scientific research indicates the need for revision of current Doppler ultrasound standards and parameters, particularly for patients receiving endovascular treatment due to renovascular hypertension. Therefore, the paper refers to the current issue of ultrasound scan interpretation in patients receiving endovascular stenting, after transplantation of kidney, as well as in pediatric patients.
\end{abstract}

\section{Introduction}

A number of important scientific reports, which significantly changed the diagnostic and therapeutic approach to the treatment of renal artery stenosis - the leading cause of secondary renal hypertension, have been published since the latest standards for ultrasound examination of renal arteries of the Polish Ultrasound Society were published (2014). The introduction of 2017 ESC Guidelines on the Diagnosis and Treatment of Peripheral Arterial Diseases (including renal arteries) significantly limited the indications for endovascular treatment, and thus angiography, which was considered to be the gold standard. As a result, a greater responsibility has been shifted to physicians performing ultrasound scanning, who are involved in first-line diagnosis of renal arterial stenosis as well as co-responsible for an optional extension of the imaging algorithm in a given patient. It should be emphasized that the so far used hemodynamic criteria for the diagnosis of renal stenosis have not changed in the official recommendations of American and European ultrasound societies (the 2013 AIUM criteria are still in effect).

A long-term follow-up in both the RENAISSANCE research program and smaller studies conducted in a group of pa- 


\section{INDICATIONS FOR DOPPLER ULTRASOUND OF RENAL ARTERIES}

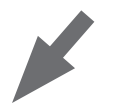

NATIVE ARTERIES (PATIENT'S OWN KIDNEY)

\section{TRANSPLANTED KIDNEY ARTERIES}

1. Hypertension (especially for a significant suspicion of renovascular hypertension)

2. Monitoring of patients with previously diagnosed renovascular disease (e.g. after endovascular intervention)

3. Vascular murmur in the epigastrium

4. Suspected vascular pathology, such as aneurysm, pseudoaneurysm, malformation or arteriovenous fistula

5. An attempt to identify the causes of renal failure in patients at risk of renal vascular disease

6. Evaluation of renal vascularization in patients with clinically known aortic dissection; traumatic patients; or those with other diseases potentially reducing kidney perfusion

7. Significant asymmetry in kidney size
1. Screening aimed at determining the baseline value of renal hemodynamic parameters

2. Follow-up of abnormalities from previous examination

3. Coexistence of tenderness, pyrexia, sepsis or disturbing clinical symptoms (increased creatinine, oliguria/anuria)

4. Evaluation of renal vascular patency

5. An attempt to identify the causes of hematuria, hydronephrosis, ureteral dilatation, bladder pathology

6. Verification of the presence of perirenal cisterns

7. Transplant assessment in patients with coexisting arterial hypertension

8. Assessment for iatrogenic damage/complications after biopsy

9. Assessment for lymphoproliferative disease

Fig. 1. Indications for Doppler ultrasound of renal arteries. Modified based on: AIUM Practice Parameter for the Performance of Native Renal Artery Duplex Sonography (2013) and AIUM Practice Guideline for the Performance of an Ultrasound Examination of Solid-Organ Transplants (2014)

tients treated with endovascular stenting showed that the use of standard diagnostic criteria for renal arterial stenosis results in an overdiagnosis of the disease. Although the results of these studies are not yet reflected in the current, though outdated, global diagnostic guidelines, we decided to present them in this paper to show the newly emerging trends.

\section{Indications for US examination}

Importantly, the indications for Doppler imaging of renal arteries have not changed (Fig. 1). Suspected correctable vascular pathology or defect is the main indication for Doppler examination both in native arteries, as well as in the arteries in a transplanted kidney. At the same time, there are no absolute contraindications to duplex sonography ${ }^{(1,2)}$.

It should be kept in mind that the examination may pose difficulty and may have limited diagnostic value in the case of obese patients, lack of respiratory cooperation and extensive reflection artifacts due to bowel gases. Ultrasonog- raphy is also of limited diagnostic value in renal parenchymal diseases ${ }^{(3,4)}$.

Limitations:

1) anatomy and congenital defects - mobile kidney, multiple renal arteries, horseshoe kidney;

2) severe condition of the patient - lack of respiratory cooperation;

3) difficult scanning conditions - large amount of intestinal gases or obesity

\section{Equipment}

Ultrasound machine intended for renal artery examination needs to be equipped with duplex function for the evaluation of color coded blood flow (preferably with additional options for visualizing low-velocity blood flow), and recording of the blood flow spectrum. Due to the deep location of both renal arteries and the kidneys themselves, a typical convex transducer with a frequency of $2-5 \mathrm{MHz}$ (usually $3.5 \mathrm{MHz}$ ) is used in most cases. In well-prepared, 
slim patients, blood flow assessment may be performed using a 6-12 MHz multi-frequency linear transducer, allowing for more accurate hemodynamic measurements and a more favorable Doppler insonation angle ${ }^{(5,6)}$.

\section{Patient preparation}

As in the case of any other ultrasonographic examination of abdominal organs, patients should report fasted, i.e. after about 8-hour fasting (the last meal should be consumed before 6 p.m. the day before the scheduled ultrasound). The use of tobacco products and chewing gums on the day of scanning is also strongly discouraged due to the risk of ingestion of large amounts of air, which promotes the formation of artifacts that affect the full diagnostic assessment. Simethicone at a dose of $80 \mathrm{mg}$ ( 2 capsules) 3 times daily 1-2 days before scanning and $80 \mathrm{mg}$ ( 2 capsules) on the day of ultrasound examination is increasingly recommended in obese and immobilized patients with impaired peristalsis, and, increasingly often, in slim patients ${ }^{(7)}$.

\section{Technique}

The aim of scanning is to visualize all renal arteries throughout their course from the aorta to the level of segmental and interlobar arteries. Additionally, the size of kidneys (in at least two dimensions), the renal corticomedullary differentiation, and the presence of focal lesions

\begin{tabular}{|c|}
\hline $\begin{array}{l}\text { Normal blood flow characteristics } \\
\text { in renal artery trunks }{ }^{(5)}\end{array}$ \\
\hline $\begin{array}{l}\text { - steep velocity curve in the contraction phase } \\
\text { - spectral window present } \\
\text { - absence of reversal blood flow direction } \\
\text { - slow return to the end-diastolic velocity } \\
\text { - flow velocity in the trunk usually the same or slightly slower than } \\
\text { in the aorta }\end{array}$ \\
\hline $\begin{array}{l}\text { Normal values of blood flow parameters } \\
\text { in renal arteries }{ }^{(1,2)}\end{array}$ \\
\hline $\begin{array}{l}\text { - PSV in the trunk of about } 100 \mathrm{~cm} / \mathrm{s} \\
\text { - RAR (renal aortic ratio, the ratio of maximum blood flow velocity } \\
\text { in the renal artery to the maximum velocity in the aorta) } \\
\text { of about } 0.8-1.0 \\
\text { - } \mathrm{Al} \text { (acceleration index, the rate of acceleration specifying the slope } \\
\text { of the curve expressed in } \mathrm{m} / \mathrm{s} 2 \text { ) } \geq 3 \mathrm{~m} / \mathrm{s}^{2} \\
\text { - AT (acceleration time, the time counted in seconds since } \\
\text { the beginning of the systolic phase to reaching the maximum } \\
\text { velocity in the mid-systolic phase) } \leq 0.07 \mathrm{~s} \\
\text { - } \mathrm{PI} \text { (pulsatility index) } 0.78-1.33 \\
\text { - RI (resistance index) about } 0.5-0.8\end{array}$ \\
\hline
\end{tabular}

Tab. 1. Blood flow characteristics in normal renal artery. For transplanted renal artery, the renal iliac ratio (RIR) is used instead of RAR (normal RIR values are identical to RAR) or hypotonia of the collective system should be always assessed. For transplanted kidneys, it is important to search for potential perirenal fluid collections that may indicate a pathological process or improper surgical technique ${ }^{(5,6)}$.

The scanning always begins with the morphological assessment of kidneys and renal vessels in B-mode images; followed by a color-coded evaluation of blood flow, with a particular attention paid to the so-called aliasing regions, which are indicative of turbulent flow, and thus a possible stenosis or arteriovenous fistula ${ }^{(1,2,5,6)}$.

Periaortic segments of renal arteries are best evaluated in the transverse plane (perpendicular to the aorta), moving from the midline to the midclavicular line in a patient in a supine position. It should be noted that renal arteries arise from the aorta, slightly below (about $1.5-2 \mathrm{~cm}$ ) the superior mesenteric artery, with the right renal artery arising from its anteriolateral aspect, and the left renal artery arising slightly lower, from its posterior-lateral aspect. Visualization of the left renal vein makes it easier to identify the left renal artery; the artery is located slightly deeper than the vein. The right renal artery is noticeably longer and runs from the posterior to the right renal vein and inferior vena cava; both venous structures may hinder diagnostic color-coded presentation and appropriate spectral recording. It may happen that despite optimal visualization of arterial trunks on B-mode and color-coded images, as well as attempts to tilt the transducer, it is impossible to obtain diagnostic values of Doppler insonation angle. In such cases, scanning in a longitudinal plane in a patient placed in the left decubitus position - the socalled "banana peel view", should be attempted. Evaluation of perihilar and intrahilar segments is performed in a patient in decubitus position with the opposite upper limb pulled up high behind the head and full extension of the opposite lower limb; for the right side - in the anterior or middle axillary line, while for the left side - in the middle or posterior axillary line $\mathrm{e}^{(5,6)}$.

Spectral recording of blood flow is the final stage of ultrasonographic assessment. Arterial vascularization is assessed in the para-aortic segment (for transplanted kidneys - at and around the anastomosis with the iliac artery), central and perihilar segment of the renal artery trunk, as well as at the intrarenal level, at least at three levels: upper pole, central part and lower pole of the kidney. While obtaining appropriate insonation angle $\left(<60^{\circ}\right)$ at the level of the main renal artery trunk plays a key role for the interpretation of hemodynamic parameters, angle correction is not needed at the intrarenal level if no assessment of the AI parameter (acceleration index) is performed ${ }^{(5-7)}$.

Blood flow velocity measurements are performed in a longitudinal projection/longitudinal section of the vessel, with the gate positioned at the central part of the stream, which, under normal conditions, corresponds to the centre of the vessel. The size of the gate should be $1 / 2-1 / 3$ of an unobstructed blood vessel lumen (1.5-2.0 $\mathrm{mm}$ in practice).

Doppler spectral samples should be recorded during patient's inhalation when the scanning is performed in the 
decubitus position or during breath-hold, when the transducer is applied in the midline; therefore patient's respiratory cooperation is of key importance ${ }^{(6)}$.

\section{Ultrasonographic measurements}

Knowledge on the nature of blood flow and standard blood flow parameters for healthy vessels is crucial in the diagnosis of renal arterial stenosis. The spectrum of blood flow velocity in renal arteries and their branches is a low resistance one. A decrease in blood flow velocity is observed in renal arterial branches while moving toward the distal direction. However, the low-resistance nature of spectrum remains unchanged (Tab. 1).

The first two of these blood flow parameters, i.e. the maximum systolic velocity and renal-aortic ratio (RAR), are evaluated in the renal artery trunk, while the other ones, i.e. acceleration rate, acceleration time and the values of vascular resistance (pulsatility index [PI] and resistivity index [RI]) - in the intrarenal branches of renal arteries. The maximum blood flow velocity in the renal arteries should always be considered in relation to the velocity of blood flow in the abdominal aorta, where the range of the maximum blood flow velocities varies from $30 \mathrm{~cm} / \mathrm{s}$ in patients with circulatory failure or major atherosclerotic lesions to $150 \mathrm{~cm} / \mathrm{s}$ in young people with hyperkinetic circulation.

In pediatric patients, it should be remembered that normal RI ranges between 0.6 and 0.8 in children under the age of 1 year ${ }^{(8,9)}$. RI values typical for adults are achieved already 6 to 12 months after birth and, according to some sources, RI values above 0.7 are not found in healthy children over 36 months of $\operatorname{life}^{(9,10)}$. Reports indicating that renal arterial stenosis cannot be excluded in children merely based on ultrasonography, with classical angiography still representing a reference method for the assessment of this patient population, are also of great importance ${ }^{(11)}$.

Velocity measurements are performed when the Doppler angle of insonation is $60^{\circ}$ or less. Measuring velocities in tortuous vascular segments, where physiological increase in velocity is always recorded, should be avoided. It is usually very difficult to confirm or rule out stenosis in this location. In such situations, the morphological image of the vessel should be carefully analyzed and measurements should be made with a wide sampling gate.

Below are presented individual blood flow parameters used in the diagnosis of significant stenosis (Tab. 2).

The RAR index compares blood flow at the site of potential stenosis with a normal reference site, i.e. with blood flow values in the abdominal aorta at the level of renal arterial origin. It must be borne in mind that if the PSV values in the abdominal aorta are abnormal (beyond the range of 40-100 $\mathrm{cm} / \mathrm{s}$ ), the calculation of the RAR index becomes useless ${ }^{(12)}$. Furthermore, PSV values in the renal artery trunk $>1.8 \mathrm{~m} / \mathrm{s}$ and $<2.0 \mathrm{~m} / \mathrm{s}$ are indicative of $<60 \%$ stenosis. RAR ratio $>3.0$ and $<3.5$ indicates a similar degree of stenosis ${ }^{(12)}$.

$\begin{gathered}\text { The diagnosis of hemodynamically } \\ \text { significant renal artery stenosis } \\ (\geq 60 \%)^{(1,2,6)}\end{gathered}$
- peak systolic velocity (PSV) $\geq 2.0 \mathrm{~m} / \mathrm{s}$
- end-diastolic velocity (EDV) $\geq 1.5 \mathrm{~m} / \mathrm{s}$
- RAR $\geq 3.5$
- acceleration time (AT) $\geq 0.08 \mathrm{~s}$
- acceleration index (Al) $<3 \mathrm{~m} / \mathrm{s}^{2}$
- RI difference $>0.05$ (stenosis occurs when there is lower RI value)
- difference in the size of the kidneys $>15 \mathrm{~mm}$

Tab. 2. Hemodynamic exponents of significant renal artery stenosis. For transplanted renal artery, the renal iliac ratio (RIR) is assessed instead of RAR. Furthermore, a comparison of RI values and the size of kidneys is not used in this group of patients

The first three of these blood flow parameters, i.e. maximum systolic velocity, end-diastolic velocity and RAR are evaluated in the renal artery trunk, while the other parameters, i.e. acceleration rate, acceleration time and vascular resistance index - in the intrarenal branches of renal arteries.

The following parameters are crucial in the diagnosis of stenosis: PSV at the stenotic site, RAR and the acceleration time in the intrarenal segment.

Long-term monitoring in patients after renal arterial angioplasty with stent implantation indicates overdiagnosis of restenosis when using the above mentioned conventional hemodynamic ultrasound criteria ${ }^{(13-15)}$. Slightly modified criteria for the diagnosis of renal in-stent restenosis proposed in the RENAISSANCE study (Tab. 3) have shown satisfactory, high sensitivity and specificity $(83.3 \%$ and $91.7 \%$, respectively) ${ }^{(13)}$; therefore, a critical look should be particularly given to the diagnosis of minor/borderline stenosis in this population.

Renal artery occlusion can be detected only when there is no evidence of the blood flow in the visualized renal artery trunk. By no means does the absence of a renal artery image allow for the diagnosis of its obstruction.

\section{Description and photo documentation of the test}

Each description of a renal artery examination should contain the following data:

- patient's name, age and, optionally, PESEL number (Polish personal identification number);

- the date of the test;

- name of the apparatus, information about the type/types of transducers and their frequencies.

The description should provide information on the location, size and morphological image of the kidneys, as 


\begin{tabular}{|l|}
\hline \multicolumn{1}{|c|}{$\begin{array}{c}\text { Restenosis criteria according } \\
\text { to RENAISSANCE }\end{array}$} \\
\hline - renal/aortic ratio (RAR) $\geq 3.5$ \\
- peak systolic velocity $(\mathrm{PSV}) \geq 225 \mathrm{~cm} / \mathrm{s}$ \\
- turbulent blood flow immediately after the stent \\
\hline
\end{tabular}

Tab. 3. Ultrasonographic morphological criteria and hemodynamic diagnosis of in-stent restenosis proposed by the authors of the RENAISSANCE study

well as details concerning the segments of the visualized renal arteries, e.g.: "The right renal artery was visualized throughout its entire course and its intrarenal branching. The left renal artery trunk was not visualized in the initial and middle section; only the perihilar section and intrarenal branching were visualized".

The description will depend on whether the imaging reveals normal or pathological renal arteries.

If the image of the renal arteries is normal, individual blood flow parameters in the trunk or intrarenal branches should not be included in the description (the photographic documentation contains images of blood vessels along with the recorded velocities).

In the event of diagnosing a pathology, details on the abnormal course and possible dilation of the renal artery should be included in the description, e.g.: "Right renal artery tortuous", "Aneurysm of the left renal artery in the hilar area- dimensions..." If atherosclerotic plaques are present, their location should be reported. If the plaques cause stenosis and increased blood flow velocity, the description should include the following values: PSV at the site of stenosis, RAR index, systolic acceleration time (AT) and the RI index - evaluated intrarenally. Stenosis should be graded as significant, i.e. $\geq 60 \%$ and eligible for treatment, or non-significant, requiring a follow-up or verification using other diagnostic tools, such as CT angiography. If no renal artery/arteries are visualized in the examination, this information should be included in the description and other imaging techniques (MRI angiography, CTangiography) should be recommended.

Photographic documentation (printouts from a video printer or a computer printer or a $\mathrm{CD}$ ) with the name and surname of the patient should be included in the documentation. The documentation consists of the images of the right and left kidney along with the size.

For normal image of renal arteries:

- a record of the spectrum and velocity measurement in the periaortic segment of the renal artery - bilaterally;

- a record of the spectrum and velocity measurement in the perihilar section of the renal artery - bilaterally;

- a record of the spectrum and the measurement of the velocity, AT and RI in intrarenal branches - bilaterally (only one photo of each side).

For stenotic arteries:

- a record of the spectrum and the measurement of the acceleration at the stenotic site;

- a record of the spectrum and the measurement of the velocity in the abdominal aorta at the exit level of renal arteries;

- a record of the spectrum, the measurement of AT and RI and velocity values in intrarenal branches;

- the image of stenosis with the use of the color coded blood flow.

For other morphological changes:

- an image e.g. of the tortuous segment of the renal artery or an aneurysm.

\section{Conclusions}

The latest guidelines of the European Society of Cardiology (2017) emphasize the role of Doppler ultrasonography as the primary, first-line examination in patients with suspected renovascular hypertension. Although previous hemodynamic criteria for the diagnosis of renal arterial stenosis proposed by global ultrasound societies have not changed significantly since 2014, current reports in line with Evidence Based Medicine point to the need for diagnostic vigilance, especially in patients with renal arterial stent, whose increased PSV may reflect physiological adaptation to the new anatomical conditions.

\section{Conflict of interest}

The authors do not report any financial or personal connections with other persons or organizations, which might negatively affect the contents of this publication and/or claim authorship rights to this publication.

\section{References}

1. American Institute of Ultrasound in Medicine, American College of Radiology, Society for Pediatric Radiology, Society of Radiologists in Ultrasound: AIUM practice guideline for the performance of native renal artery duplex sonography. J Ultrasound Med 2013; 32: 1331-1340.

2. American Institute of Ultrasound in Medicine, American College of Radiology, Society for Pediatric Radiology, Society of Radiologists in Ultrasound: AIUM practice guideline for the performance of an ultrasound examination of solid-organ transplants. J Ultrasound Med 2014; 33: $1309-1320$.

3. Granata A, Zanoli L, Clementi S, Fatuzzo P, Di Nicolò P, Fiorini F: Resistive intrarenal index: myth or reality? Br J Radiol 2014; 87: 20140004.

4. Eklöf H, Ahlström H, Magnusson A, Andersson LG, Andrén B, Hägg A et al.: A prospective comparison of duplex ultrasonography, captopril renography, MRA, and CTA in assessing renal artery stenosis. Acta Radiol 2006; 47: 764-774.

5. Cochlin DL: Vascular disorders of the kidney. In: Allan PL, Baxter GM, Weston MJ (eds.): Clinical Ultrasound. Elsevier, Amsterdam 2011: 467485 . 
6. Jakubowski W: Standardy badań ultrasonograficznych Polskiego Towarzystwa Ultrasonograficznego. Roztoczańska Szkoła Ultrasonografii, Warszawa-Zamość 2008.

7. Pellerito JS, Polak JF (eds.): Introduction to Vascular Ultrasonography. Saunders, Philadelphia 2012.

8. Coley BD: Pediatric applications of abdominal vascular Doppler. Part II. Pediatr Radiol 2004; 34: 772-786.

9. Bude RO, DiPietro MA, Platt JF, Rubin JM, Miesowicz S, Lundquist C: Age dependency of the renal resistive index in healthy children. Radiology 1992; 184: 469-473.

10. Murat A, Akarsu S, Ozdemir H, Yildirim H, Kalender O: Renal resistive index in healthy children. Eur J Radiol 2005; 53: 67-71.

11. Trautmann A, Roebuck DJ, McLaren CA, Brennan E, Marks SD, Tullus K: Non-invasive imaging cannot replace formal angiography in the diagnosis of renovascular hypertension. Pediatr Nephrol 2017; 32: 495-502.

12. Zierler RE (ed.): Strandness. Obrazowanie dopplerowskie w chorobach naczyń. Medipage, Warszawa 2013.

13. Rocha-Singh K, Jaff MR, Lynne Kelley E, RENAISSANCE Trial Investigators: Renal artery stenting with noninvasive duplex ultrasound follow-up: 3-year results from the RENAISSANCE renal stent trial. Catheter Cardiovasc Interv 2008; 72: 853-862.

14. Bakker J, Beutler JJ, Elgersma OE, de Lange EE, de Kort GA, Beek FJ: Duplex ultrasonography in assessing restenosis of renal artery stents. Cardiovasc Intervent Radiol 1999; 22: 475-480.

15. Napoli V, Pinto S, Bargellini I, Vignali C, Cioni R, Petruzzi P et al.: Duplex ultrasonographic study of the renal arteries before and after renal artery stenting. Eur Radiol 2002; 12: 796-803. 